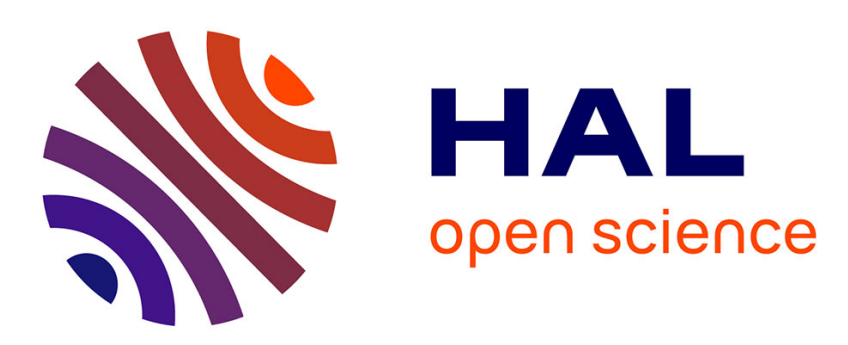

\title{
Realistic model of spine geometry in the human skeleton in the Vicon system
}

Miroslawa M. Dlugosz, Wieslaw Chwala, Pawel Maciejasz, Witold Alda

\section{To cite this version:}

Miroslawa M. Dlugosz, Wieslaw Chwala, Pawel Maciejasz, Witold Alda. Realistic model of spine geometry in the human skeleton in the Vicon system. Bio-Algorithms and Med-Systems, 2012, 8, pp.123-132. 10.2478/bams-2012-0007 . lirmm-00830436

\section{HAL Id: lirmm-00830436 \\ https://hal-lirmm.ccsd.cnrs.fr/lirmm-00830436}

Submitted on 22 Feb 2021

HAL is a multi-disciplinary open access archive for the deposit and dissemination of scientific research documents, whether they are published or not. The documents may come from teaching and research institutions in France or abroad, or from public or private research centers.
L'archive ouverte pluridisciplinaire $\mathbf{H A L}$, est destinée au dépôt et à la diffusion de documents scientifiques de niveau recherche, publiés ou non, émanant des établissements d'enseignement et de recherche français ou étrangers, des laboratoires publics ou privés. 
Bio-Algorithms and Med-Systems 8(1),2012, pp

CJagiellonian University, Medical College, Kraków, Poland

doi: 10.2478/bams-2012-0007

DE DE GRUYTER

VERSITA

\title{
REALISTIC MODEL OF SPINE GEOMETRY IN THE HUMAN SKELETON IN THE VICON SYSTEM
}

\author{
Mirosława DŁugosz ${ }^{1}$, WiesŁaw ChWaŁA², PaweŁ Maciejasz ${ }^{3}$, \\ WITOLD ALDA ${ }^{4}$ \\ ${ }^{1}$ A GH University of Science and Technology, Faculty of Electrical Engineering, \\ Automatics, Computer Science and Electronics, Department of Automatics, Krakow, \\ ${ }^{2}$ University School of Physical Education in Krakow, \\ ${ }^{3}$ Institute of Precision and Biomedical Engineering, Warsaw University \\ of Technology, \\ ${ }^{4}$ A GH University of Science and Technology, Faculty of Electrical Engineering, \\ Automatics, Computer Science and Electronics, Department of Computer Science, \\ Krakow
}

\begin{abstract}
The human spine is definitively one of the most important parts of a living body. From biomechanical point of view, this organ is the most complicated structure, and a dynamic analysis of its motion still requires more detailed models be created. The aim of this study is to build an accurate computer model of the geometry of the spine and insert it into available skeleton models, used in Vicon. Up to now such models were simplified and that is why they were not sufficient for research of detailed motion of the spine.

The paper describes the process of creating models of the vertebrae. During research, authors simultaneously used two techniques: $3 \mathrm{D}$ scanning of the vertebrae and computed modeling in 3D graphics software. A universal data format: .obj used to keep the information about surface shape of an object, its colour, texture etc. is presented in detail. In a discussion on Vicon data formats and relations between them, several file types are taken under consideration: marker files: . $m k r$, global model parameters files: .mp, files of the model structure: . $m o d$. But first of all, step-by-step instructions of how to connect the files with geometrical objects for the visualization purposes, are presented.
\end{abstract}

KEYWORDS: VICON system, motion capture, spine modeling, biomechanics 


\section{Introduction}

Human spine is definitively one of the most important parts of a living body. Because of its important functions as well as a number of emerging pathologies (such as scoliosis) or injuries (e.g. of cervical spine in different types of accidents), the spine has been a subject of a comprehensive research for many years now, both experimental and modeling. In this paper we focus on mathematical and computational models of the spine, which can be built for different purposes and analyzed in a few aspects: kinematic [1], dynamic [2], investigating mechanical properties with Finite Element Method [3], etc. From biomechanical point of view, this organ represents the most complicated structure, thus the analysis of its motion and dynamics still requires more detailed models to be created. These models should cover both geometry of vertebrae, as well as kinematics and dynamics of the entire spine.

The purpose of this paper is to build an accurate computer model of the geometry of the spine and combine it with an already existing skeleton model offered by the Vicon system (www.vicon.com), which is well established solution in motion capture and biomechanical analysis. Considering geometry only, makes our goal rather modest, still not trivial. In spite of its simplicity the results can be very useful and helpful for doctors during various orthopedic treatment.

Among techniques for motion tracking and analysis, the best and most popular is undoubtedly a motion capture approach. Vicon, which is used in this work, belongs to such systems. It measures and registers motion trajectories of selected points on patient's body. In the system under consideration we use passive, reflective markers fixed to a human body. Their motion is recorded by a set of calibrated infrared video cameras. For the professional use, the system offered by the Vicon company is currently considered to be a leader of this method. It includes software for $3 \mathrm{D}$ motion reconstruction, analysis and visualization.

Models of complete or partial human body (containing muscular and skeletal systems) used in Vicon, are rather simplified. They are usually sufficient for visualization and analysis of motion of the limbs, but they definitely contain not enough information for detailed investigation of motion of the spine. A precise model of the spine is mostly needed by orthopedists and physiotherapists who use Vicon system for diagnosis of scoliosis as well as for other pathologies.

We have already proposed the computational model for Vicon system, which allows to calculate the exact positions and orientations of each vertebra during movement [4]. While the verification of our model still remains indispensable for further study, in this work we extend the model with the possibility to realistically visualize each vertebra. The paper describes the 
process of creating 3D representation of the vertebrae which can be used to visualize motion of the spine calculated using Vicon software. During the research, the authors simultaneously used two techniques: 3D scanning of the vertebrae and computed modeling using 3D graphics software.

A universal data format: .obj [5] (created by Alias-Wavefront company) has been used to keep the information about a shape of an object's surface, its color, texture etc. Its general structure is presented later.

In a discussion on Vicon data formats and relations between them, several file types have been taken under consideration: marker files - .mkr, global model parameters files - .mp, files of the model structure - .mod. But first of all, step-by-step instructions of how to connect the files with geometrical objects for the visualization purposes, are presented.

\section{Vicon system}

Vicon system is to some extent in the center of our research, cause all motion capture data are obtained and analyzed with its help. Main ideas and capabilities, as well as general structure, are presented in this section.

Vicon is a complete system, which contains hardware devices with all the harness, as well as the necessary software. The hardware includes cameras, which work on the edge of visible and infrared frequencies. The resolution of the cameras ranges from 1megapixel up to 16 megapixels, while a very high throughput is up to $250 \mathrm{fps}$ over gigabit Ethernet. It enables high quality recordings of a very fast motion. Still the most important part of the system is sophisticated software. Most important programs are: the Vicon Workstation which gathers motion information from the markers attached to a body, Vicon BodyBuilder which allows building biomechanical body models using a special BodyLanguage, and Vicon Polygon to make reports, perform motion analysis and visualization.

\section{File formats and their purpose}

Several file formats are used in the course of building the final model. They are shortly presented in this section. We start from brief description of a very popular in 3D modeling, general purpose, obj format developed by AliasWavefront Company. It defines geometry of a 3D object by specifying its surface approximated using a mesh of polygons - usually triangles or quadrangles. Any .obj file must contain a list of vertexes with their $x, y, z$ Cartesian coordinates, usually followed by a list of faces described using vertex numbers. Vertices and faces may be grouped into objects, in our case in the way that a single bone or a group of bones (e.g. head and pelvis cases) 
are marked as separate objects and labeled with unique names. These names have to match relevant objects defined in .mod and.$m k r$ files. We limit ourselves to a simplified form of a file, however full specification of.$o b j$ format is quite rich. It may contain information about normal vectors attached to vertexes, coordinates of textures mapped on faces, parameters defining smoothing of the mesh e.g. by using parametric curves such as Bezier and Bsplines, rendering attributes, and many others.

The next $c 3 d$ (Coordinate 3D) format is a biomechanics standard, currently maintained by Motion Lab Systems, Inc. from Baton Rouge, USA. This is a binary format, which contains time trajectories of every marker attached to the body and registered by the cameras during motion. To be more specific, the $c 3 d$ file contains numerical data of every sample, i.e. its $x, y, z$ coordinates with optional information such as measurement accuracy or camera parameters. Additionally to those numerical data, there can be attached so called analogous data acquired from other sensors, such as force plates, etc. There is also a possibility to add metadata for description purposes. Raw information from a $c 3 d$ file can be displayed as a set of marker images (Figure 1). Also results of the performed analysis may be added to this file as scalar or vector values changing over time.

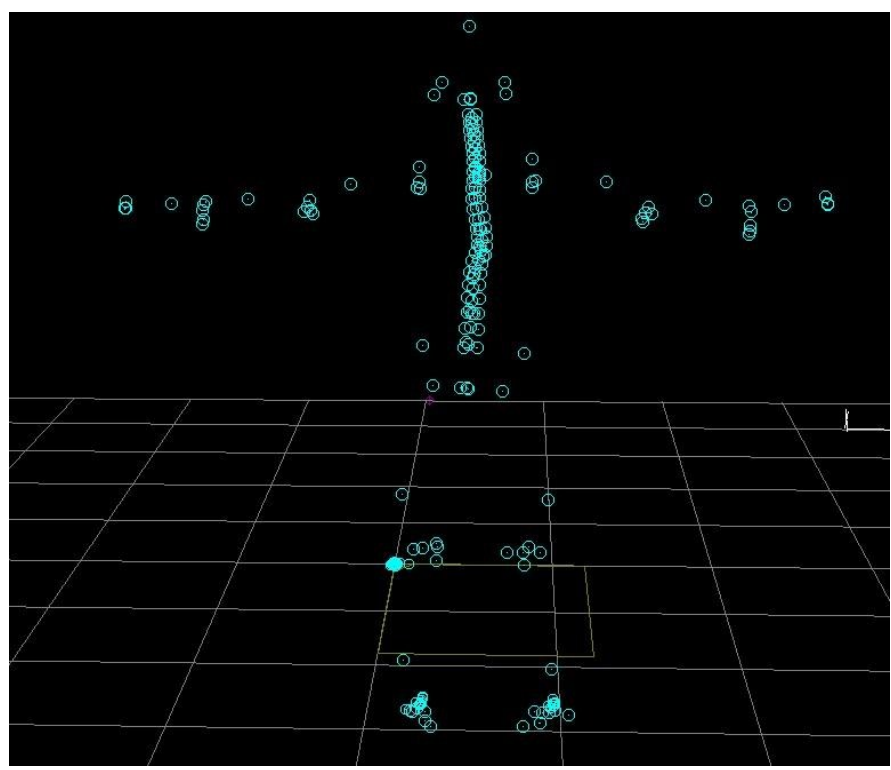

Figure 1. Positions of markers in single time instance extracted from $c 3 d$ file. 
Following formats are bound to Vicon Systems only. The model file (.mod) describes the structure as well as kinetics and kinematics of complex moving objects (e.g. human body) and is the most important, but also the most sophisticated file. The body description is written in a special programming language named BodyLanguage, in a form of a script. In Vicon environment it can be read and interpreted by BodyBuilder program.

The BodyLanguage script contains 4 types of objects (in the sense of programming language objects). These are: numbers, points (composed of three numbers), segments (group of points) and rotations. Points can be either real or virtual. In the first case they represent real markers, while in the latter they result from modeling or other calculations. Virtual points are used to indicate coordinates of special points (such as joints) or add points to build a mesh which represents a surface more realistically. Besides that, both types of points are treated by BodyBuilder equally.

Segments are groups of points (both real and virtual), each of which usually represents a part of a body (pelvis, bone, etc.) which may be considered as a rigid body. It can be moved or rotated around a specific point as a whole.

Rotations are also considered as separate objects, described by three Euler angles. Rotations can represent either angular orientation of a single object in global coordinate system, or relative orientation of two segments.

New objects can be defined on the basis of existing objects as a result of expressions composed of objects, operators and functions. In addition to objects, which form a hierarchical body structure, also kinetic modeling can be applied. BodyLanguage can add mass properties to the objects, as well as forces, moments and reactions. These features are interpreted by BodyBuilder to create physically correct kinetic model.

Creating a complete working model with BodyBuilder is based on $c 3 d$ data acquired in an individual experiment. A model (.mod) file is added to these data in order to build a coherent kinetic structure of many separate measured points. Then the model is scaled by means of the parameter file (.mp), which contains anthropometric data of a patient. From a programmers point of view they are simply constant values used by a .mod file, but stored separately.

The marker (.mkr) file, which contains a list of markers, definitions of bones (as groups of markers) and connections between them, is also used for computation. Marker file is a bridge between .mod file, and corresponding .$c 3 d$ file with trajectories. 


\section{Spinal cord geometry model}

The entire geometry of the spinal cord has been assembled from separately modeled single vertebrae. Accurate modeling of their shape is quite a tedious task. We have started with 3D scanning of single vertebrae using 3D Picza LPX-250 Roland 3D scanner. It resulted with a rather complex, irregular and noisy mesh, which must have been thoroughly processed later. The artifacts in the form of sharp spikes can be seen in Figure 2 .

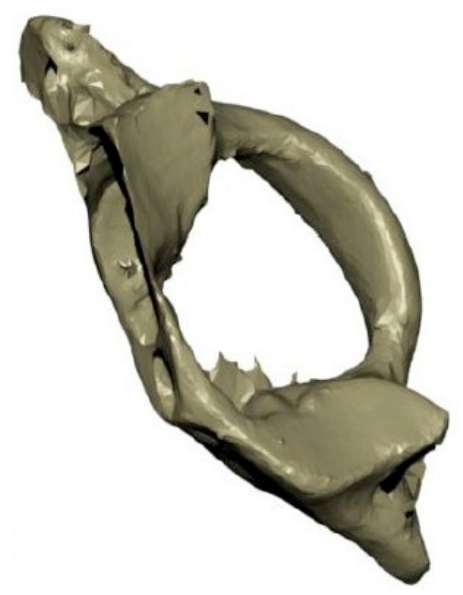

Figure 2. Model of a C1 vertebra obtained from 3D scanner.

Subsequent smoothing of the surface was done using 3D Studio Max program and it appeared to be quite labor-consuming task as well. Final adjustments of the vertebrae geometry model have been made with the usage of the Blender software. They have also been assembled into segments. Figure 3 shows a single segment in the interactive blender environment, where it can be moved, turned, bent, etc., due to attached Python script. A complete spine geometry model, ready to use in other programs is shown in Figure 4. 


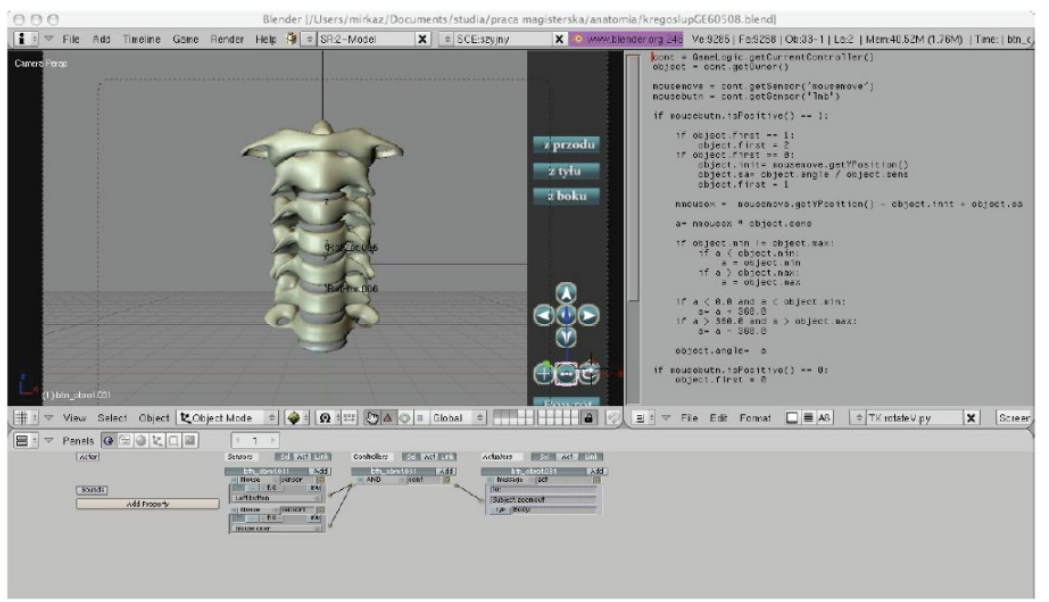

Figure 3. Model of the single spine segment assembled in Blender with Graphic User Interface added for interactivity.

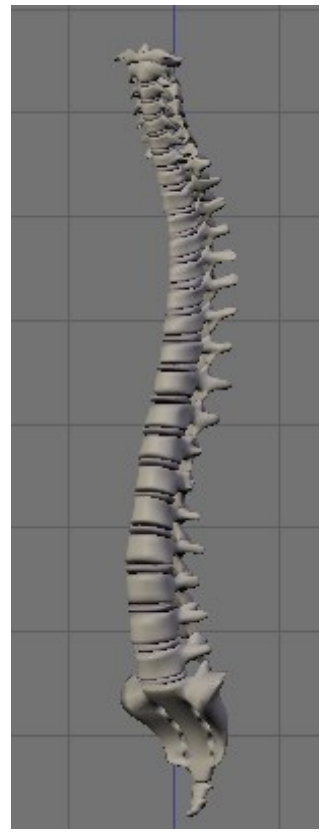

Figure 4. A complete geometry model of spine exported from Blender. 


\section{Model visualization}

Complete model of the human body as well as its part, which was created in BodyBuilder, can be imported to the subsequent Vicon's program - Polygon. Polygon application is a software tool designed to create, edit and export reports (Polygon Reports) or/and animations (Polygon Presentations). It enables reading and analyzing of motion captured data processed by Vicon Workstation and Vicon BodyBuilder applications. Originally the captured data are seen as a set of markers (Figure 1) which are subsequently used to build a correct human shape. Segments of the human skeleton can be visualized in several ways. Depending on the type of tests carried out, the user of Polygon application can choose from visualization as: segments, primitive solids such as boxes, diamonds or truncated cones (Figure 5).

This simple representation does not need any extra data beside those based on markers. However definitely much better results can be achieved using meshes, because the segments are more like real bones. Proper geometry is often needed to help in analysis and diagnosis.

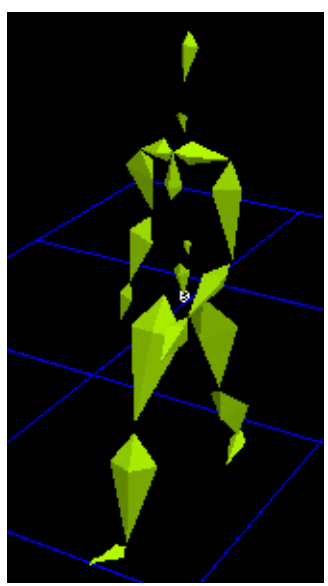

Figure 5. Human body model visualized by Polygon application. The segments are represented by so called diamonds (joined tetrahedrons).

The mesh is loaded from initially prepared .obj file described briefly earlier. Meshes form objects, and these in turn can be gathered into object groups. To display them correctly, object groups must contain the same segment names, as those indicated in corresponding . $\mathrm{mkr}$ and. $\bmod$ files. Authors in their work used the modified golembones.obj (one of standard Vicon files), which originally defined groups of all human bones and 
segments, except complete spinal cord. The spine was marked out using only two elements (Figure 6). Normally golembones model contains the thorax segment as well, but for spine analysis this group is not necessary and was deleted.

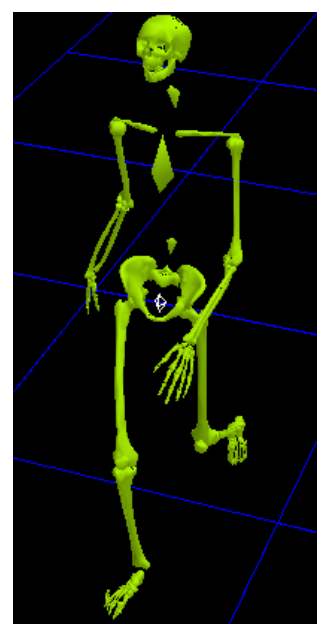

Figure 6. Incomplete human skeleton model. Spine is marked out by two solids: first indicates the position of $\mathrm{C}_{7}$ vertebra, while the second $-\mathrm{Th}_{10}$.

\section{Results and conclusions}

Finally the model of vertebrae, based on precise 3D scans has been incorporated to a complete skeleton giving a realistic image. We are convinced that this representation of the spine can be extremely helpful to doctors in their diagnostic decisions. Currently the shape of the spine is determined by spline approximation, according to [4]. In future we plan work on even more realistic approximation, as well as on continuing the development of kinematic and dynamic models. 

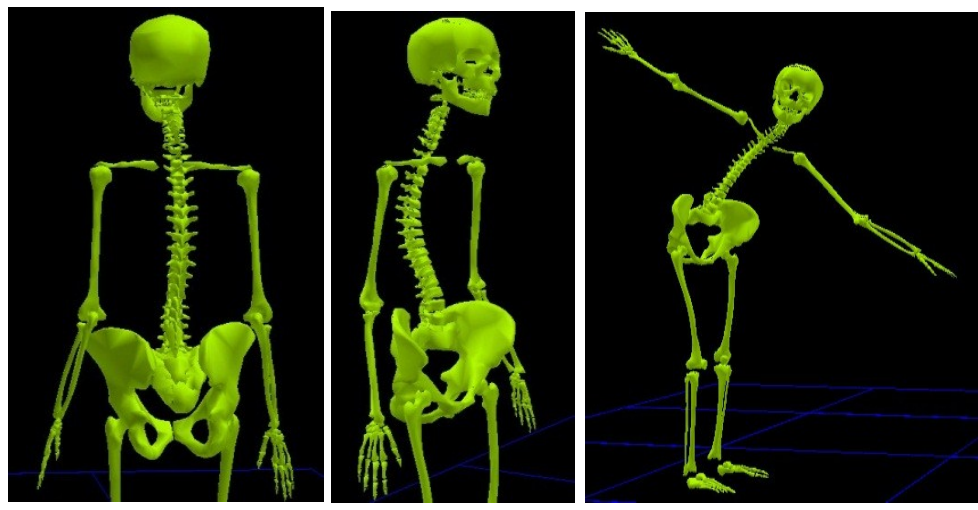

Figure 7. Model of the spine based on geometrically correct vertebrae incorporated into the skeleton

\section{Acknowledgements}

The possibility of using 3D Picza LPX-250 Roland 3D scanner for registration of all vertebrae, generously offered to us by the Computer Graphics Group from the Department of Automatics AGH is kindly acknowledged. One of us (WA) kindly acknowledges partial support from N519 443039 Polish KBN Project.

\section{Bibliography}

1. Monheit, G. Badler N.I. (1991). A Kinematic Model of the Human Spine and Torso. IEEE Computer Graphics and Applications, vol. 11(2).

2. de Jager, M. (1993). Mathematical Modelling of the Human Cervical Spine: A Survey of the Literature, Report No. WFW-93.027 April 1993, Computational and Experimental Mechanics Faculty of Mechanical Engineering Eindhoven University of Technology The Netherlands.

3. Scifert J, Totoribe K, Goel V, Huntzinger J. (2002). Spinal cord mechanics during flexion and extension of the cervical spine: a finite element study. Pain Physician, 5(4):394-400.

4. Maciejasz P, Chwala W. (2006). A method of evaluating the kinematics of a spine deformed by scoliosis using a motion capture system. Journal of Biomechanics, vol. $39, \mathrm{~S} 172$

5. .obj file specification is available on

http://www.martinreddy.net/gfx/3d/OBJ.spec 